\title{
Expanding the application of a UV-visible reporter for transient gene expression and stable transformation in plants
}

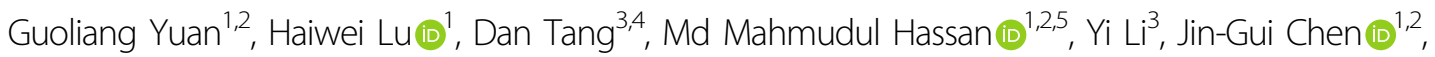 \\ Gerald A. Tuskan ${ }^{1,2 凶}$ and Xiaohan Yang (10 ${ }^{1,2 \bowtie}$
}

\begin{abstract}
Green fluorescent protein (GFP) has been widely used for monitoring gene expression and protein localization in diverse organisms. However, highly sensitive imaging equipment, like fluorescence microscope, is usually required for the visualization of GFP, limitings its application to fixed locations in samples. A reporter that can be visualized in realtime regardless the shape, size and location of the target samples will increase the flexibility and efficiency of research work. Here, we report the application of a GFP-like protein, called eYGFPuv, in both transient expression and stable transformation, in two herbaceous plant species (Arabidopsis and tobacco) and two woody plant species (poplar and citrus). We observed bright fluorescence under UV light in all of the four plant species without any effects on plant growth or development. eYGFPuv was shown to be effective for imaging transient expression in leaf and root tissues. With a focus on in vitro transformation, we demonstrated that the transgenic events expressing 1x eYGFPuv could be easily identified visually during the callus stage and the shoot stage, enabling early and efficient selection of transformants. Furthermore, whole-plant level visualization of eYGFPuv revealed its ubiquitous stability in transgenic plants. In addition, our transformation experiments showed that eYGFPuv can also be used to select transgenic plants without antibiotics. This work demonstrates the feasibility of utilizing 1x eYGFPuv in studies of gene expression and plant transformation in diverse plants.
\end{abstract}

\section{Introduction}

Reporter genes and systems have been playing essential roles in biological sciences. Various reporter genes have been developed and adapted to a diverse set of organisms, e.g., lac $Z$ in microbes ${ }^{1}, \beta$-glucuronidase (GUS) in plants $^{2}$, luciferase $(\mathrm{LUC})^{3}$ and green fluorescent protein $(\mathrm{GFP})^{4}$ in both prokaryotic and eukaryotic organisms, to name a few. The application of one or another reporter is mainly dependent on the organism of interest and the

\footnotetext{
Correspondence: Gerald A. Tuskan (tuskanga@ornl.gov) or

Xiaohan Yang (yangx@ornl.gov)

'Biosciences Division, Oak Ridge National Laboratory, Oak Ridge, TN 37831, USA

${ }^{2}$ The Center for Bioenergy Innovation, Oak Ridge National Laboratory, Oak Ridge, TN 37831, USA

Full list of author information is available at the end of the article

These authors contributed equally: Guoliang Yuan, Haiwei Lu, Dan Tang
}

availability of the imaging and detection technologies. In plants, GUS, LUC, and GFP are extensively used as the reporters or selectable markers. Technically, GUS, with the presence of glucuronides, and LUC, with the presence of luciferin, can be visualized by naked eyes or under light microscopy (e.g., a luminometer or modified optical microscope), respectively, requiring specific substrates. In contrast, GFP simply requires a fluorescence microscope with no need for specific substrates. Recently, a new reporter, $R U B Y$, was developed without the need of using special equipment or substrates ${ }^{5}$. $R U B Y$, generating the red pigment betalain, is directly visible by naked eyes. However, the red pigment in the leaf or other tissue is irreversible once it is produced, which may inevitably interfere with biological processes, such as photosynthesis.

\section{(c) The Author(s) 2021}

(c) (i) Open Access This article is licensed under a Creative Commons Attribution 4.0 International License, which permits use, sharing, adaptation, distribution and reproduction cc) in any medium or format, as long as you give appropriate credit to the original author(s) and the source, provide a link to the Creative Commons license, and indicate if changes were made. The images or other third party material in this article are included in the article's Creative Commons license, unless indicated otherwise in a credit line to the material. If material is not included in the article's Creative Commons license and your intended use is not permitted by statutory regulation or exceeds the permitted use, you will need to obtain permission directly from the copyright holder. To view a copy of this license, visit http://creativecommons.org/licenses/by/4.0/. 
In contrast, GFPuv (a GFP variant) was optimized for maximal fluorescence to be observed by naked eyes under UV light instead of using fluorescence microscope. Although multiple GFPuv-related studies have been reported in plants, its applications have been limited. GFPuv has been used for the quantification of the fluorescence of GFPuv-labeled plant pathogens and the indication of gene expression in model species tobacco ${ }^{6-9}$.

Agrobacterium-mediated in planta transformation and tissue-culture-based transformation are the most commonly used methods for plant genetic engineering ${ }^{10-13}$. However, one unavoidable issue related to tissue-culturebased transformation and in planta transformation with antibiotic resistance genes as selectable markers is falsepositive transformant shoots or escapees on selection media ${ }^{14,15}$. Consequently, efforts to eliminate falsepositive transformants, typically by using GUS assay and PCR analysis of target DNAs, have been deployed. Recently, a novel version of GFPuv, called eYGFPuv which is a mutant of an enhanced yellow GFP-like protein derived from Chiridius poppei, was identified and used to generate brilliant green, fluorescent flowers in petunia ${ }^{16,17}$. Positive transgenic shoots were identified under $\mathrm{UV}$ light after incorporating an eYGFPuv gene construct $^{17}$. However, this approach requires a large $7.5 \mathrm{~kb}$ expression cassette, which can create an obstacle during vector construction and cloning, particularly when multiple genes or viral delivery approaches are required. To address this limitation, we reduced the eYGFPuv expression cassette by using $1 \mathrm{x}$ eYGFPuv ( $2 \mathrm{~kb}$ in length) instead of $3 \mathrm{x}$ eYGFPuv (Fig. 1A) and explored the potential application of $1 \mathrm{x}$ eYGFPuv in transient expression and stable transgenics in Arabidopsis, tobacco, poplar (an important perennial woody bioenergy and landscape plant ${ }^{18}$ ) and citrus (an important woody fruit $\mathrm{crop}^{19}$ ).

\section{Results}

Transient expression of eYGFPuv in leaf and root tissues

To achieve strong green florescence during plant transformation, we placed $1 \mathrm{x}$ eYGFPuv under the control of a cauliflower mosaic virus (CaMV) 35S promoter, a COR47-5'-UTR enhancer ${ }^{20}$ and a HSPT878 terminator ${ }^{21}$ to increase the expression level of eYGFPuv. We then tested the quality of the eYGFPuv plasmid through protoplast transformation in Arabidopsis and included a 35S: GFP plasmid as a control. Both GFP and eYGFPuv showed strong green fluorescence under fluorescence microscope, though the fluorescence inensity of eYGFPuv was relatively weaker than that of GFP under same setting (Fig. 1B). As expected, vivid green fluorescence was observed by naked eyes in the Eppendorf tube containing eYGFPuv-expressing protoplasts under UV light, but not in the GFP-expressing protoplasts (Fig. 1B). Next, we tested the activity of eYGFPuv using Agrobacteriummediated leaf infiltration in Nicotiana benthamiana. Strong fluorescent signal was observed on the leaf under fluorescence microscope three days post infiltration (Fig. 1C). Next, we examined the infiltrated leaf following the procedure illustrated in Fig. 2A and Supplemental Video 1. As expected, vivid green fluorescence was exhibited on the leaf, along with a red autofluorescence background, under UV light (Fig. 2B). These observations

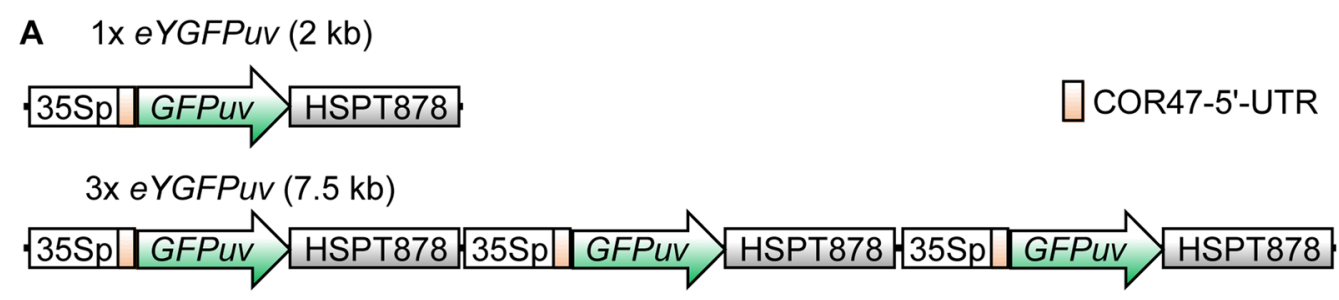

B

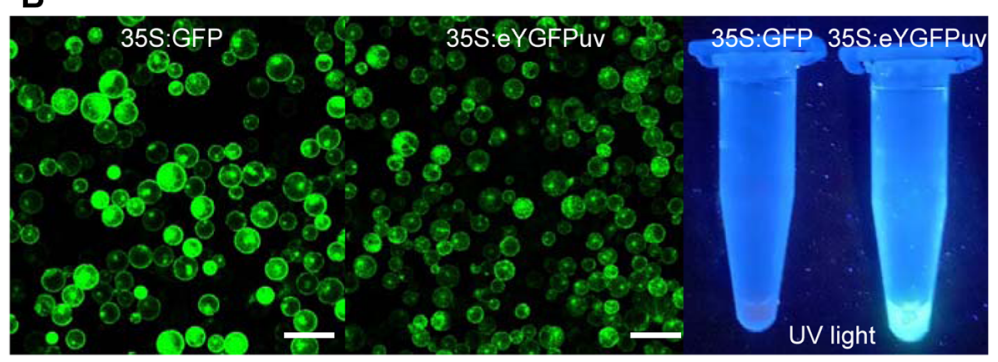

C

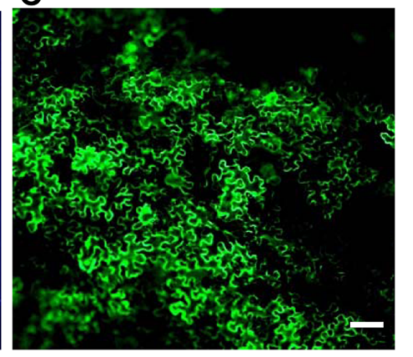

Fig. 1 Transient expression of eYGFPuv in the Arabidopsis protoplasts and the tobacco leaf. A Illustration of $1 \times$ eYGFPUv and $3 \times$ eYGFPUv. B Visualization of GFP-expression of 35S:GFP and 35S:eYGFPuv in Arabidopsis protoplasts under fluorescence microscope and UV light, respectively (Scale bar, $100 \mu \mathrm{m}$ ). C Visualization of eYGFPuv expression of 35S:eYGFPuv under fluorescence microscope, three-day post tobacco ( $\mathrm{N}$. benthamiana) leaf infiltration (Scale bar, $50 \mu \mathrm{m}$ ) 


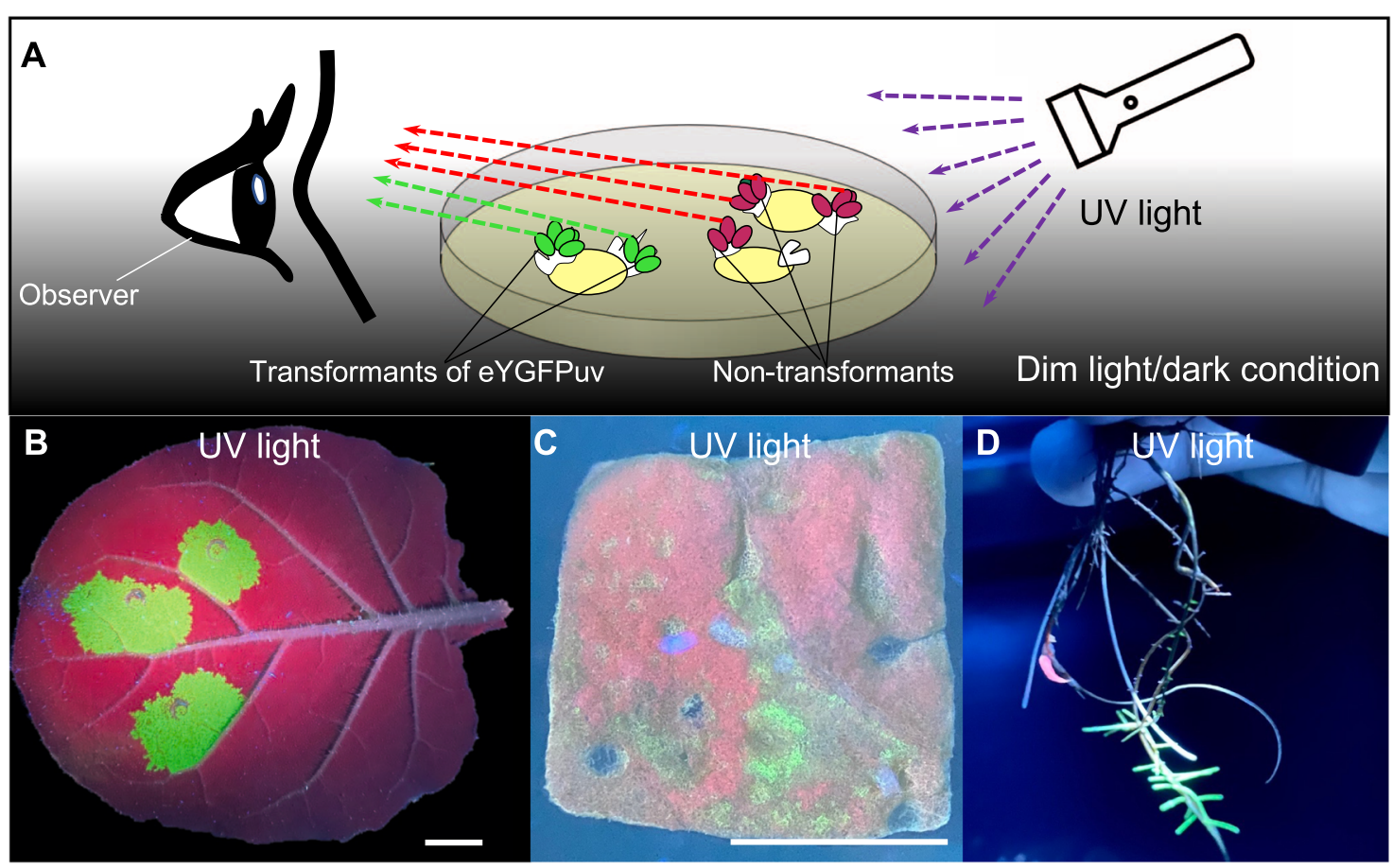

Fig. 2 Visualization of eYGFPuv in leaf and root during transient expression. A Illustration of visualizing eYGFPuv-expressing samples using UV light. Under long-wavelength UV light (365 nm), eYGFPuv-expressing leaves show green fluorescence, while non-transformed leaves show red autofluorescence. B Visualization of eYGFPuv in leaf under UV light, three days post tobacco ( $N$. benthamiana) leaf infiltration. Scale bar, $1 \mathrm{~cm}$. C Visualization of eYGFPuv in leaf disk of tobacco ( $N$. tabacum 'Xanthi') under UV light, one week post Agrobacterium-mediated in vitro transformation. Scale bar, $1 \mathrm{~cm}$. D Visualization of eYGFPuv in the root under UV light, three weeks post poplar root transformation

suggest that $1 \mathrm{x}$ eYGFPuv can generate fluorescence strong enough for detecting eYGFPuv expression during transient expression at both cell and tissue levels. We then examined the expression of eYGFPuv using tobacco ( $N$. tabacum 'Xanthi') leaf disc transformation. The green fluorescence was observed under the UV light on the leaf discs seven days after infection (Fig. 2C). These results demonstrate that eYGFPuv can be easily visualized both in leaf infiltration and leaf disc transformation.

Because of the wide application of root transformation in producing transformants for assessing gene function, particularly in species recalcitrant to A. tumefaciens-mediated transformation $^{22,23}$, we evaluated if $1 \mathrm{x}$ eYGFPuv could be used for easy and rapid identification of transformed roots. We transformed the 1x eYGFPuv expression cassette into the hybrid poplar clone 717 (Populus tremula $\times P$. albaclone INRA 717-1B4; hereafter '717') using A. rhizogenes. We were able to observe green fluorescence signal in roots as early as 14 days post A. rhizogenes inoculation. After three weeks, we moved rooted plants to sterile water to allow further root growth and development. Approximately four weeks after inoculation, we were able to distinguish transgenic roots from non-transgenic roots with the aid of the strong eYGFPuv expression (Fig. 2D). Our results suggest that $1 \mathrm{x}$ eYGFPuv can serve as a reliable marker for selecting transgenic roots without the need of fluorescence microscope and quantifying transformation efficiency in root transformation systems.

\section{Supplementary Video 1. Visualization of eYGFPuv- expressing events}

Vivid green fluorescence was observed in the eYGFPuvexpressing poplar callus excited by a UV light.

\section{eYGFPuv assisted early selection during tissue culture- based plant transformation}

Tissue culture-dependent plant transformation is an indispensable and widely used method for plant research. To examine 1x eYGFPuv expression throughout organogenesis, we initiated $A$. tumefaciens-mediated transformation in herbaceous plant tobacco ( $N$. tabacum 'Xanthi'), woody plants poplar clone 717 and citrus rootstock 'Carrizo'. In tobacco, calli and small shoots with clear bright green fluorescence were observed two weeks post Agrobacterium infection, thus facilitating early selection of transformants (Fig. 3A).

In comparison with herbaceous plants, the transformation of woody plants is usually more challenging and time consuming. Thus, based on the transgenic tobacco results, we speculate that $1 \mathrm{x}$ eYGFPuv could play a useful role in 


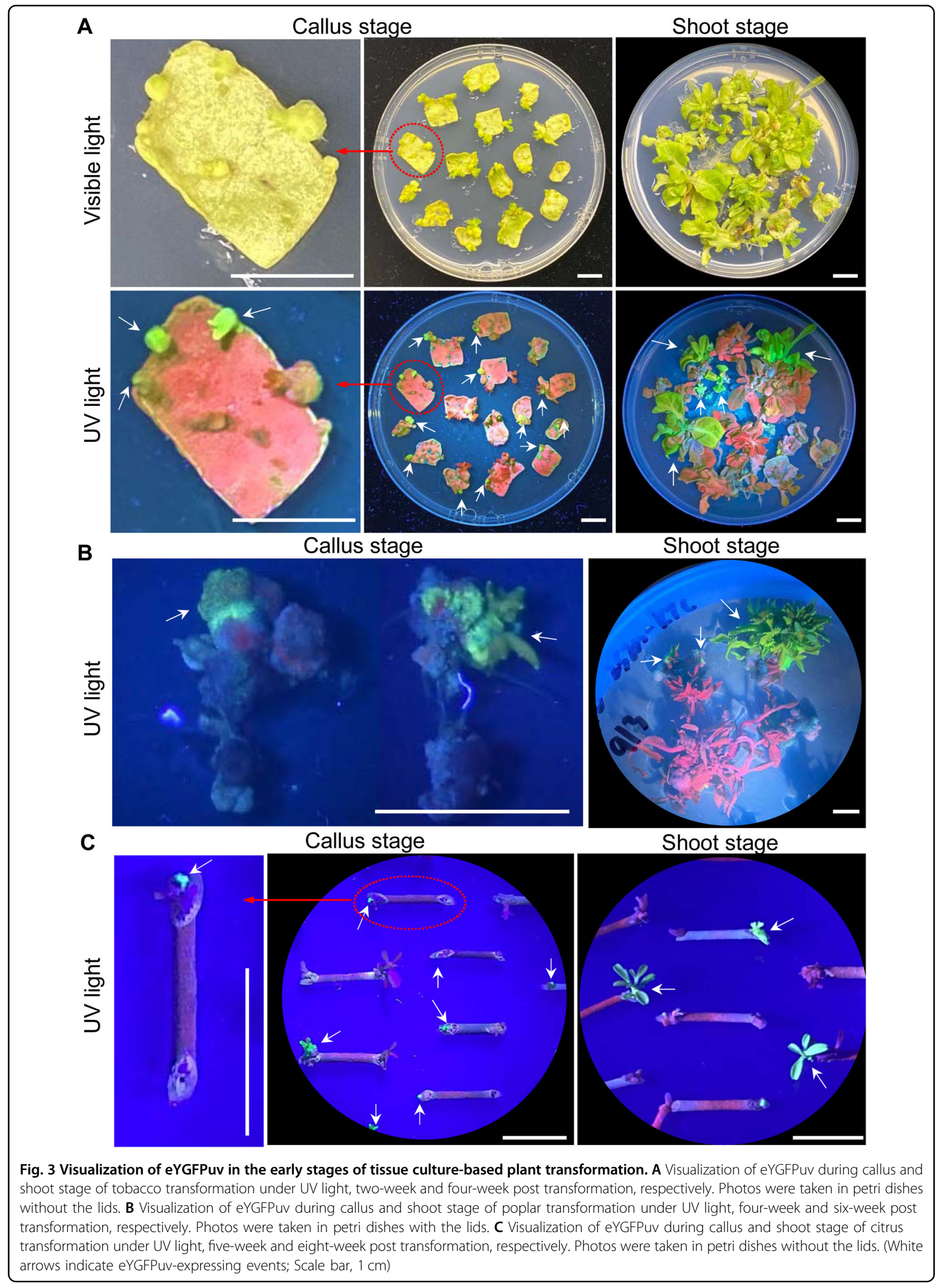




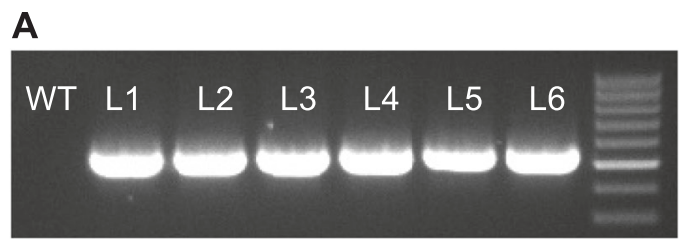

B

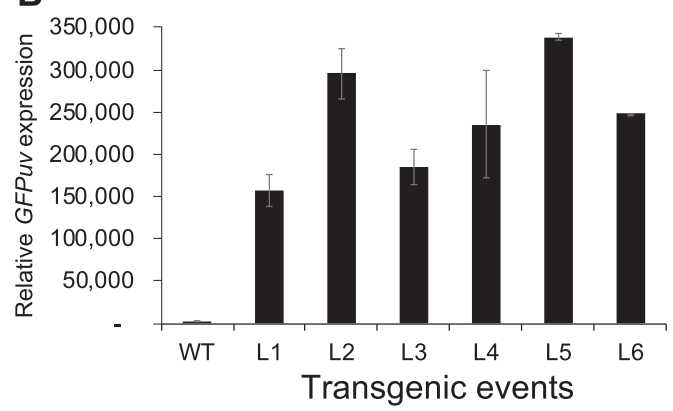

Fig. 4 Analysis of eYGFPuv transformants of hybrid poplar clone 717. A Genotyping of eYGFPuv transgenic lines. B RT-qPCR analysis of eYGFPuv expression level in the transformants and the WT plants. There was no detectable signal in the WT plant sample. The background noise of WT was normalized as 1. Error bars denote standard error over three technical replicates

early selection of woody plant transformants. Regrettably, we were not able to detect any eYGFPuv signal during the first three-week of callus induction stage in poplar. However, we did observe eYGFPuv-expressing calli and shoots after transferring callus tissue onto shoot induction medium and introducing them to light (Fig. 3B). Hereafter, the eYGFPuv signal maintained at an easily detectable level. Expression of eYGFPuv enabled us to select positive transgenic events under UV light by week five to six post transfection, as opposed to traditional PCR-based genotyping at 10 12 weeks post infection. Ubsequent PCRbased genotyping confirmed the insertion of the eYGFPuv gene into the genome in eYGFPuv positive events (Fig. 4A). Interestingly, quantitative reverse transcription PCR (RTqPCR) revealed a maximum of two-fold difference in the expression level of the eYGFPuv gene among six transgenic events that showed similarly eYGFPuv intensity under UV light (Fig. 4B). Citrus transgenic callus and shoots showed similar bright green fluorescence under UV light, while untransformed explants and regenerated tissues showed red autofluorescence (Fig. 3C). Our observations indicate that $1 \mathrm{x}$ eYGFPuv expression was stable during organogenesis in tobacco, poplar and citrus, and could facilitate the early selection of transgenic callus and shoots.

\section{Whole-plant level visualization of eYGFPuv in transgenic plants}

In tobacco, eYGFPuv positive shoots were transferred to rooting medium at six weeks post infection. Compared with the red autofluorescence of the wild-type (WT) tobacco, petioles, stems and roots of the transgenic plants showed a uniform and bright green fluorescence with weaker green signal on the leaf (Fig. 5A). In poplar, eYGFPuv-expressing shoots were transferred to rooting medium $\sim 12$ weeks post infection. In contrast to the red autofluorescence in wild type, bright green fluorescence was observed in the whole-plant level of eYGFPuv transformants, including leaves, stems and roots, under UV light (Fig. 5B). Similar to tobacco and poplar, in citrus the whole plant containing $1 \mathrm{x} e Y G F P u v$ appeared vivid green after rooting (Fig. 5C). The easily detectable feature of $1 \mathrm{x}$ eYGFPuv at the whole-plant level facilitates quick selection of transformed plants grown both in tissueculture media in the growth chamber and in soil in the greenhouse (Fig. 5D).

Finally, we studied the performance of $1 \mathrm{x} e Y G F P u v$ in multiple generations of transformed Arabidopsis. The T1 plants of transgenic Arabidopsis were obtained using floral dip, one of the most commonly used Agrobacterium-mediated in planta methods. eYGFPuv-expressing T1 seedlings were easily recognized under UV lamp and 87.5\% $(n=40)$ kanamycin-resistant seedlings displayed eYGFPuv signals (Fig. 6A). It is highly possible that most of the plants displaying strong eYGFPuv signals contain single-copy transgene because single-copy T-DNA insertion is predominant in Agrobaterium-mediated transformation $^{24,25}$. Indeed, one line with strong eYGFPuv signals contains a single-copy transgene, as indicated by a segregation ratio of 1:2.93 $(n=212)$ in T2 generation. Therefore a single copy transgene insertion is adequate for eYGFPuv visualization. This easily detectable feature enables the use of eYGFPuv for antibiotic-free selection of transformants (Fig. 6B). We then monitored eYGFPuv during both vegetative and reproductive growth. In comparison with WT plants, brilliant green fluorescence was observed in rosette leaves, inflorescences, siliques and petals of T1 and T2 transgenic lines without any visible detrimental effects on plant growth and development (Fig. 5E). eYGFPuv positive lines were also confirmed by PCR-based genotyping.

\section{Discussion}

In this study, we demonstrate that green fluorescence can be seen under UV light by naked eyes at both the tissue (e.g., leaf, root) and the whole-plant level through transient gene expression and stable transformation of $1 \mathrm{x}$ eYGFPuv in herbaceous and woody plants. UV-excitable eYGFPuv can be easily imaged at a wide range of scales from the sub-meter level seedlings to whole plants without need for emission filters. Another advantage of eYGFPuv as a reporter is that the eYGFPuv-expressing tissue or whole plants can be directly visualized in a petridish or tissue culture vessel without removing the lids 

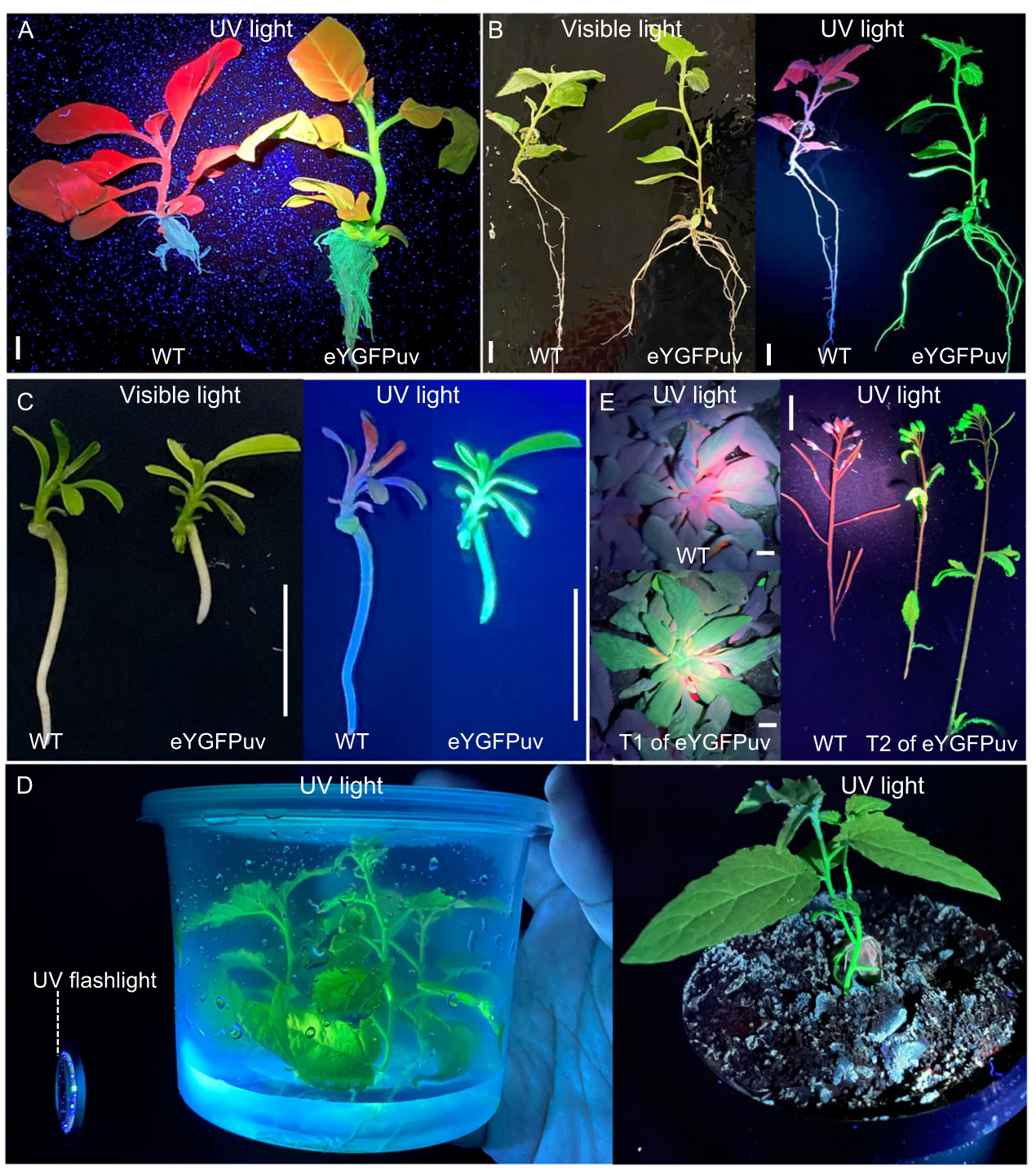

Fig. 5 Visualization of eYGFPuv in whole-plant level in different species. A Visualization of eYGFPuv in rooted plants of tobacco under UV light, 10 weeks post transformation. B Visualization of eYGFPuv in rooted plant of poplar under UV light, three months post transformation. C Visualization of eYGFPuv in rooted plant of citrus under UV light, 14 weeks post transformation. D Visualization of eYGFPuv in poplar plants placed in medium and soil under UV light, more than three months post transformation. E Visualization of eYGFPuv in T1 and T2 of Arabidopsis transgenic plants after inplanta transformation under UV light. (Scale bar, $1 \mathrm{~cm}$ )

(Supplemental Video 1 and Fig. 5D). And eYGFPuv signals can be seen in dim light or dark condition though dark condition (e.g., dark box or dark room) is usually necessary for high-quality imaging. These features suggest that $1 \mathrm{x}$ eYGFPuv has a wide range of applications in plant science research, as illustrated in Fig. 7.

In general, selectable markers play an important role in plant transformation ${ }^{13}$. In the in vitro transformation, $1 \mathrm{x}$ eYGFPuv can be used as a selectable marker for in vivo selection of transformants through live imaging without the need for destructive sampling required by PCR-based genotyping or GUS staining, saving time and cost (Fig. 7A). To our knowledge, false-negative transformation error is usually not an issue for plant transformation. It is very common that the reporter, selectable marker or trait genes do not express or express at low level in some transformants due to the well-known "position effect". These falsenegative transformants are usually not selected for further analysis. In contrast, false-positive transformation error caused by antibiotic resistance-based selection is not avoidable. The false-positive transformants can be minimized with the eYGFPuv reporter during plant transformation. Recently, it was reported that transgenic and geneedited shoots were created through de novo meristem induction without the need for tissue culture and a luciferase reporter was used to indicate the delivery of 


\section{A}

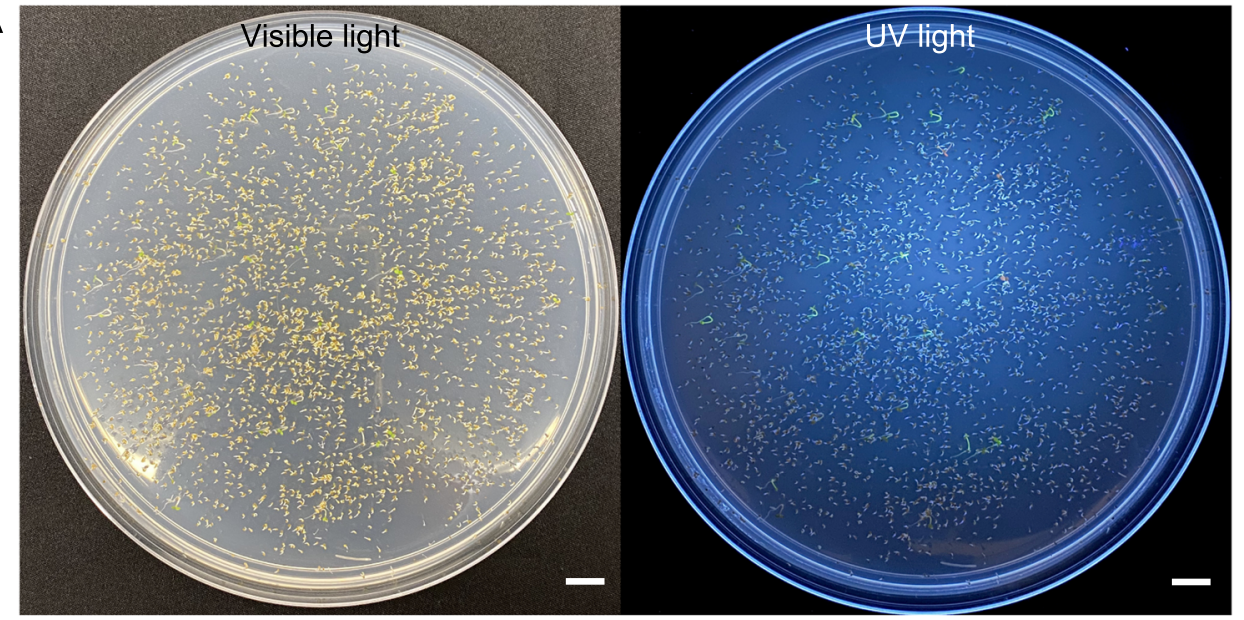

B

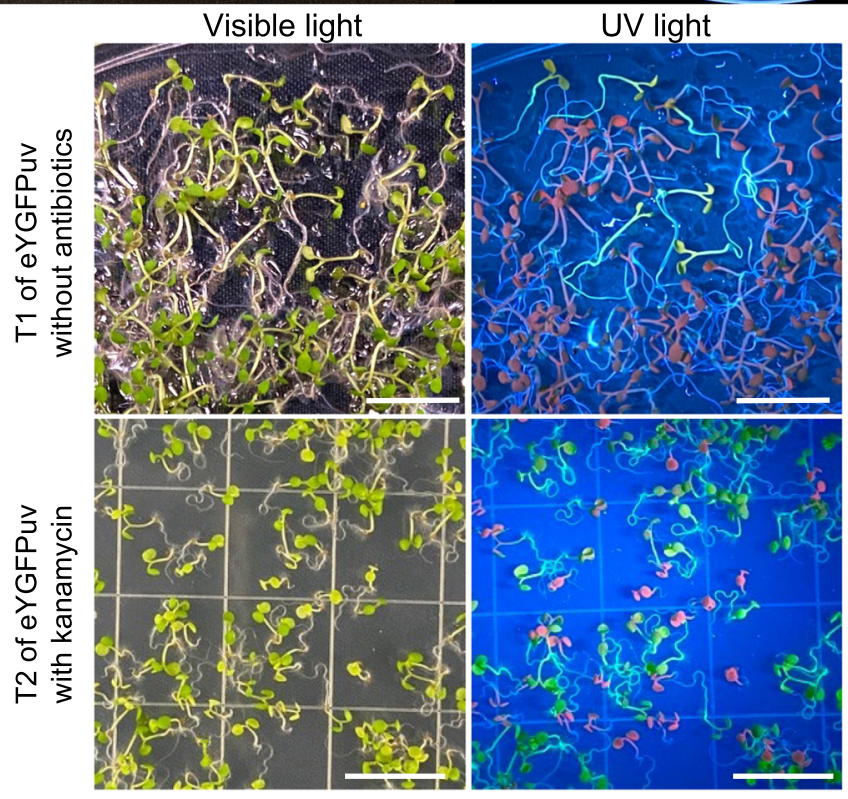

Fig. 6 eYGFPuv assisted selection in Arabidopsis. A Germination of T1 seeds on kanamycin selection plate. B Germination of T1 seeds on plant MS plate without antibiotics and germination of $\mathrm{T} 2$ seeds on kanamycin selection plate. (Scale bar, $1 \mathrm{~cm}$ )

transgenes ${ }^{26}$. The application of $1 \mathrm{x}$ eYGFPuv during in planta transformation could sidestep the need for substrate and luciferase imaging system and identify the transgenic shoots in real-time (Fig. 7B). Over the past years, the presence of antibiotics in genetically modified plants has raised concerns regarding possible risks for human health and the environment ${ }^{27}$. Here we have shown that $1 \mathrm{x}$ eYGFPuv can be used for antibiotic-free selection in Arabidopsis after flora dip based transformation (Fig. 6B). Similarly, we believe that eYGFPuv can be used for antibiotic-free selection in any other plant species, which can be transformed through floral dip, e.g., Brassica napus ${ }^{28}$. It is expected that eYGFPuv can be used as a visual selectable marker for antibiotic-free in vitro transformation (Fig. 7C). Based on the observations in poplar transformation, it is apparent that eYGFPuv-expressing transgenic plants can be easily visualized and tracked in the growth chamber, greenhouse or field (Figs. 5D and 7D), which is particularly useful for the long-term maintenance and evaluation of genetically modified plants. Furthermore, the expression of target genes can be tracked through protein-fusion with an eYGFPuv $^{29}$ (Fig. 7D).

Determination of promoter activity is critical for plant functional genomics and plant synthetic biology research ${ }^{30,31}$. The methods of studying promoter activity are usually based on the expression of a reporter gene driven by the promoter of interest. As a robust reporter, eYGFPuv can be utilized for characterizing promoter properties, such as tissue specificity of a promotor, hormonal regulation of gene expression, synthetic promoter screening and the tightness/leakiness of an inducible promoter (Fig. 7E). In addition, eYGFPuv-based biosensor 


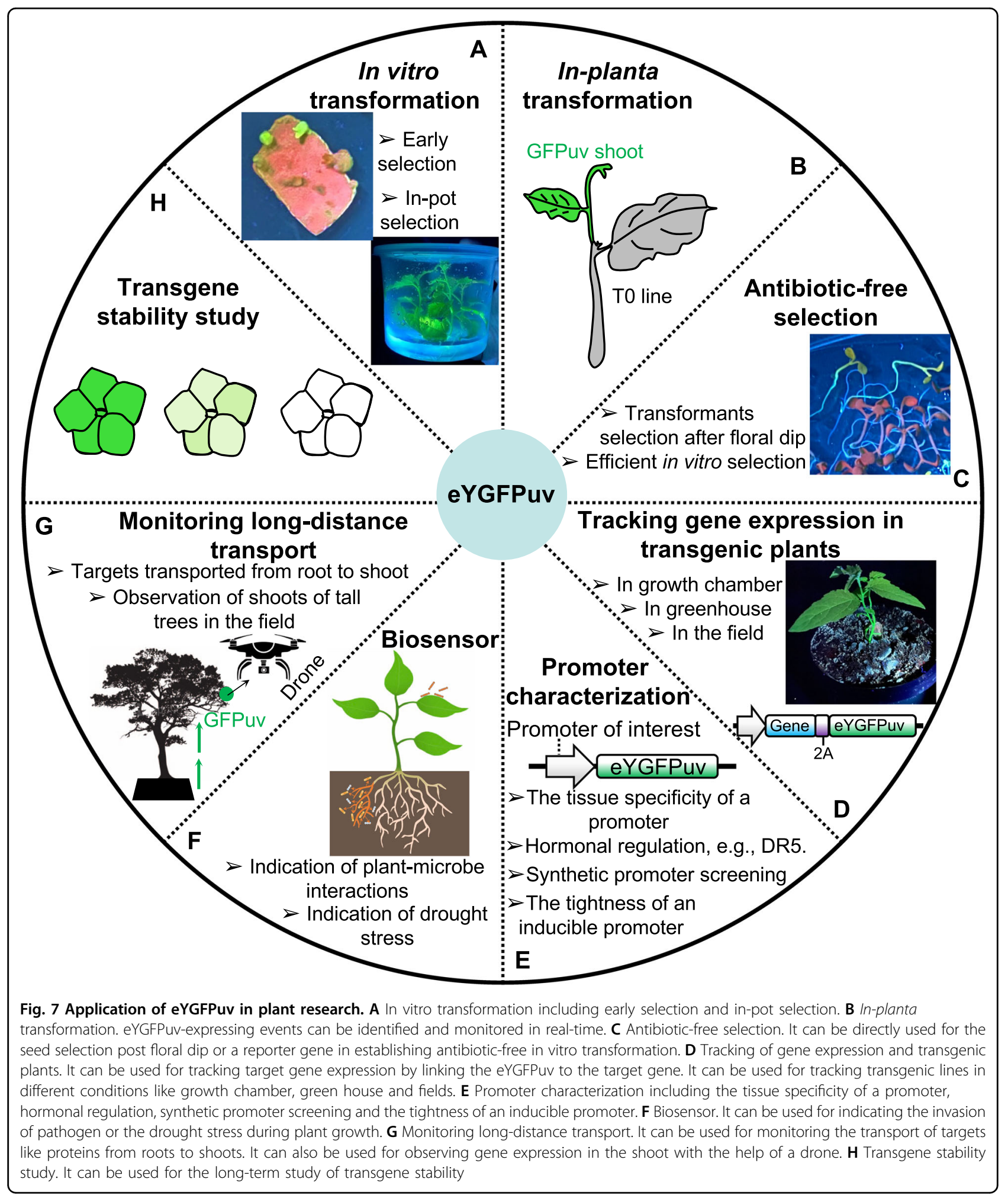

system can be used for the indication of plant-microbe interactions ${ }^{6-8}$, drought stress and other environmentally triggered reactions (Fig. $7 \mathrm{~F}$ ). We speculate that $1 \mathrm{x}$ eYGFPuv can serve as a monitor of the long-distance transport of proteins through the plant vascular system ${ }^{32}$. Also, it can be used for monitoring gene expression in the shoots of tall trees in combination with unmanned aerial surveillance (Fig. 7G). Finally, given that the stability of 
transgene expression is a challenge for plant genetic engineering $^{33}$, eYGFPuv appears suitable for laboratory and field tests over multiple generations for assessing the long-term expression stability of transgenes (Fig. $7 \mathrm{H}$ ). Furthermore, the eYGFPuv-based biosensor has the potential of enabling advanced plant biotechnology and synthetic biology by combining fluorescent reporters and plant imaging ${ }^{34}$. However, as a new reporter gene, some characteristics of eYGFPuv still remain unclear, e.g., the stability of eGFPuv mRNA and protein in the transgenic plants, such as multiple generations of annual plants or multiple years of perennial plants. The potential of eYGFPuv need to be further exploited in the future.

\section{Materials and methods Construction of vector}

To build the $1 \mathrm{x}$ eYGFPuv expression vector, two gBlocks gene fragments containing enhancer COR47-5'UTR, eYGFPuv and terminator HSPT878 were synthesized from Integrated DNA Technologies (Coralville, Iowa). The gBlocks were assembled into pGFPGUSPlus (Addgene plasmid \# 64401) using the NEBuilder HiFi DNA Assembly Cloning Kit (New England BioLabs, Catalog \#E5520S). The presence of eYGFPuv, and its promoter and terminator sequences in the binary vector, was confirmed by Sanger sequencing.

\section{Arabidopsis protoplast transformation}

The isolation and transformation of Arabidopsis protoplasts was performed as described previously ${ }^{35}$.

\section{Arabidopsis transformation}

The Agrobacterium tumefaciens strain 'GV3101' was used for the transformation of Arabidopsis thaliana wild type 'Col-0' by the floral dip method with modification as described by Yuan et. $\mathrm{al}^{36}$.

\section{Tobacco leaf infiltration}

Infiltration of tobacco ( $N$. benthamiana) was performed as described by $\mathrm{Li}^{37}$.

\section{Tobacco transformation}

The transformation of tobacco ( $N$. tabacum 'Xanthi') was performed based on a previously described method ${ }^{38}$. In short, after three days of co-cultivation with the Agrobacterium, the explants were transferred to the selection medium (MS solid medium with $2 \mathrm{mg} / \mathrm{L} 6-\mathrm{BA}, 200 \mathrm{mg} / \mathrm{L}$ Timentin and $100 \mathrm{mg} / \mathrm{L}$ kanamycin or $10 \mathrm{mg} / \mathrm{L}$ hygromycin). The eYGFPuv positive shoots were transferred to rooting medium (MS basal medium with $200 \mathrm{mg} / \mathrm{L}$ Timentin).

\section{Poplar transformation}

The eYGFPuv plasmid was transformed into A. tumefaciens strain 'GV3101' using electroporation and then transformed into the hybrid poplar clone 717 (Populus tremula $\times$ P. alba clone INRA 717-1B4) following a published method ${ }^{39}$. Throughout the organogenesis stage, kanamycin $(50 \mathrm{mg} / \mathrm{L})$ was used to select transgenic events and Timentin $(200 \mathrm{mg} / \mathrm{L})$ and cefotaxime $(300 \mathrm{mg} / \mathrm{L})$ were used to inhibit the growth of $A$. tumefaciens. eYGFPuv fluorescence was checked regularly using a $365 \mathrm{~nm}$ UV flashlight. In addition, we performed genotyping PCR and quantitative reverse transcription PCR (RT-qPCR) to confirm transformants and determine the expression level of eYGFPuv in selected transformants, respectively. After SDS-based genomic DNA extraction ${ }^{40}$, forward primer 5'-CACGGCAACCTCAACG-3' and reverse primer 5'-CTCGACACGTCTGTGGG-3' were used for genotyping PCR. After extracting total RNA from leaf samples using Spectrum ${ }^{\mathrm{TM}}$ Plant Total RNA Kit (Sigma-Aldrich, $\mathrm{MO}$ ), we checked RNA quality and quantity with a ND1000 UV-Vis Spectrophotometer (Thermo Fisher Scientific, Waltham, MA). We then treated all RNA samples with DNase (DNase I, amplification grade; Invitrogen, Carlsbad, CA) and used them for cDNA synthesis using SuperScript III Reverse (Invitrogen, Carlsbad, CA), following the manufacturer's instructions. We performed RTqPCR with three technical replicates per reaction using Platinum SYBR Green qPCR SuperMix with ROX (Invitrogen, Carlsbad, CA) on a StepOnePlus ${ }^{\mathrm{TM}}$ Real-Time PCR System (Applied Biosystems, Foster City, CA). We included two references genes, EF1-beta (Potri.009G01860) and eIF5A (Potri.018G10730), the primers of which were previously tested in several poplar species ${ }^{41-43}$. The following eYGFPuv-specific primers 5'-GAATCCAATCCACGAGT CCTT-3' and 5'-ACCTGCTGGTACTCCACTAT-3' were used. The relative gene expression was determined using the $\Delta \Delta \mathrm{Ct}$ method.

\section{Root transformation in poplar}

We used the A. rhizogenes strain 'ARqual' to produce transgenic roots in the hybrid poplar clone 717 by adapting protocol described by Qiao and Libault ${ }^{44}$. To prepare A. rhizogenes culture for root transformation, we transformed the eYGFPuv plasmid into $40 \mu \mathrm{L}$ competent cells using electroporation at $2.5 \mathrm{kV}, 200 \Omega$ and $25 \mu \mathrm{F}$ and grew the cells in $960 \mu \mathrm{L}$ liquid LB medium in an incubating shaker at $250 \mathrm{rpm}$ and $28^{\circ} \mathrm{C}$ for $1.5 \mathrm{~h}$. We then plated the $A$. rhizogenes culture on solid LB medium containing $50 \mathrm{mg} / \mathrm{L}$ kanamycin for two days at $28^{\circ} \mathrm{C}$. After performing colony PCR confirmation of the presence of the eYGFPuv plasmid, we grew an individual colony in $50 \mathrm{ml} \mathrm{LB}$ medium containing $50 \mathrm{mg} / \mathrm{L}$ kanamycin overnight. The $A$. rhizogenes cells were then pelleted by centrifuging at $4000 \mathrm{rpm}$ for $10 \mathrm{~min}$ and then resuspended in freshly prepared dilution buffer containing $3.92 \mathrm{mg} / \mathrm{L}$ acetosyringone and $1 \mathrm{~mL} / \mathrm{L}$ DMSO at an OD600 nm of 0.3-0.6. To generate roots, we collected 
four-week-old shoot tips sprouting from trimmed greenhouse poplar plants and then wounded the bottom $1.5 \mathrm{~cm}$ region of the stems with multiple fine cuts. The wounded shoot tips were then inserted into autoclaved rockwool blocks, each of which was pre-soaked with $40 \mathrm{ml}$ diluted A. rhizogenes culture. The inoculated shoot tips were grown under the condition of $22{ }^{\circ} \mathrm{C}, 70 \%$ humidity and under $16 \mathrm{~h}$ light $/ 8 \mathrm{~h}$ dark photoperiod and watered with $40 \mathrm{ml}$ sterile $\mathrm{H}_{2} \mathrm{O}$ every five to six days. About two to three weeks after inoculation, rooted shoot tips were carefully removed from individual rockwool blocks and kept in sterile $\mathrm{H}_{2} \mathrm{O}$ for future analysis.

\section{Citrus transformation}

The transformation of 'Carrizo' citrange rootstock (Citrus sinensis 'Washington' sweet orange $\times$ Poncirus trifoliata) was performed as previously described ${ }^{45}$ with some modifications. In brief, the fresh A. tumefaciens strain 'GV3101' or 'EHA105' solution was resuspended in a liquid MS medium containing $30 \mathrm{~g} / \mathrm{L}$ sucrose and $50 \mathrm{mg} / \mathrm{L}$ acetosyringone. We cut the four-week-old, etiolated epicotyls into $1 \mathrm{~cm}$ length segments and soaked them in resuspension solution for $20 \mathrm{~min}$. The explants were blotted dry on sterile filter paper and then transferred onto solid MS medium containing $50 \mathrm{mg} / \mathrm{L}$ acetosyringone. After three days of co-cultivation, we transferred the explants to MS medium containing $30 \mathrm{~g} / \mathrm{L}$ sucrose, $3.0 \mathrm{mg} /$ L 6-BA, $100 \mathrm{mg} / \mathrm{L}$ Timentin and $75 \mathrm{mg} / \mathrm{L}$ kanamycin and incubated them under a light intensity of $60 \mu \mathrm{mol} / \mathrm{m}^{2} \mathrm{~s}^{-1}$ with a $16 \mathrm{~h}$ light $/ 8 \mathrm{~h}$ dark photoperiod. All the explants were inspected for GFP fluorescence under the ongwavelength $(365 \mathrm{~nm})$ UV light. The GFP-positive shoots were transferred to a rooting medium consisting of $1 / 2 \mathrm{MS}$ medium with $30 \mathrm{~g} / \mathrm{L}$ sucrose, $0.1 \mathrm{mg} / \mathrm{L}$ NAA and $0.1 \mathrm{mg} / \mathrm{L}$ IBA, Timentin $100 \mathrm{mg} / \mathrm{L}$ and activated carbon $0.6 \mathrm{~g} / \mathrm{L}$.

\section{UV flashlight}

Different UV flashlight sources were tested in this study: LIGHTFE UV302D (365 nm) and uvBeast UVB-V3-365 $(365 \mathrm{~nm})$, which are suitable for small and big samples, respectively.

\footnotetext{
Acknowledgements

The writing of this manuscript is supported by the Center for Bioenergy Innovation (CBI), a U.S. Department of Energy (DOE) Research Center supported by the Biological and Environmental Research (BER) program and the U.S. DOE BER Genomic Science Program, as part of the Secure Ecosystem Engineering and Design (SEED) Scientific Focus Area. Oak Ridge National Laboratory is managed by UT-Battelle, LLC for the U.S. Department of Energy under Contract Number DE-AC05-000R22725.

\section{Author details}

${ }^{1}$ Biosciences Division, Oak Ridge National Laboratory, Oak Ridge, TN 37831, USA. ${ }^{2}$ The Center for Bioenergy Innovation, Oak Ridge National Laboratory, Oak Ridge, TN 37831, USA. ${ }^{3}$ Department of Plant Science and Landscape Architecture, University of Connecticut, Storrs, CT 06269, USA. ${ }^{4}$ National Center for Citrus Improvement, College of Horticulture, Hunan Agricultural University, Changsha 410128 Hunan, China. ${ }^{5}$ Department of Genetics and Plant Breeding,
}

Patuakhali Science and Technology University, Dumki, Patuakhali 8602, Bangladesh

\section{Author contributions}

G.Y. and X.Y conceived the research. G.Y., H.L., and D.T. conducted the experiments and wrote the paper with input from other authors. G.Y. and M.H. made the vectors. X.Y., Y.L., G.T., and J.C. supervised the research and revised the manuscript.

\section{Data availability}

The plasmids will be available at Addgene.

\section{Conflict of interest}

The authors declare no competing interests. This manuscript has been authored by UT-Battelle, LLC under Contract No. DE-AC05-00OR22725 with the U.S. Department of Energy. The United States Government retains and the publisher, by accepting the article for publication, acknowledges that the United States Government retains a non-exclusive, paid-up, irrevocable, worldwide license to publish or reproduce the published form of this manuscript, or allow others to do so, for United States Government purposes. The Department of Energy will provide public access to these results of federally sponsored research in accordance with the DOE Public Access Plan (http://energy.gov/downloads/doe-public-access-plan).

Supplementary information The online version contains supplementary material available at https://doi.org/10.1038/s41438-021-00663-3.

Received: 20 May 2021 Revised: 25 July 2021 Accepted: 1 August 2021 Published online: 01 November 2021

\section{References}

1. Fromont-Racine, M., Rain, J.-C. \& Legrain, P. Toward a functional analysis of the yeast genome through exhaustive two-hybrid screens. Nat. Genet. 16, 277-282 (1997).

2. Jefferson, R. A., Kavanagh, T. A. \& Bevan, M. W. Gus fusions: beta-glucuronidase as a sensitive and versatile gene fusion marker in higher plants. Embo J. $\mathbf{6}$ 3901-3907 (1987).

3. Smale, S. T. Luciferase assay. Cold Spring Harb. Protoc. 2010, pdb.prot5421 (2010).

4. Kain, S. R. et al. Green fluorescent protein as a reporter of gene-expression and protein localization. Biotechniques 19, 650-655 (1995).

5. He, Y., Zhang, T., Sun, H., Zhan, H. \& Zhao, Y. A reporter for noninvasively monitoring gene expression and plant transformation. Hortic. Res. 7, 152 (2020).

6. Fonseca, J. P. et al. Iron-sulfur cluster protein NITROGEN FIXATION S-LIKE1 and its interactor FRATAXIN function in plant immunity. Plant Physiol. 184, 1532-1548 (2020).

7. Wang, Z. et al. Whole transcriptome sequencing of Pseudomonas syringae pv. actinidiae-infected kiwifruit plants reveals species-specific interaction between long non-coding RNA and coding genes. Sci. Rep. 7, 4910 (2017).

8. Wang, K., Kang, L., Anand, A., Lazarovits, G. \& Mysore, K. S. Monitoring in planta bacterial infection at both cellular and whole-plant levels using the green fluorescent protein variant GFPuv. N. Phytol. 174, 212-223 (2007).

9. Werner, S., Breus, O., Symonenko, Y., Marillonnet, S. \& Gleba, Y. High-level recombinant protein expression in transgenic plants by using a doubleinducible viral vector. Proc. Natl Acad. Sci. USA 108, 14061-14066 (2011).

10. Gelvin, S. B. Agrobacterium-mediated plant transformation: the biology behind the "gene-jockeying" tool. Microbiol Mol. Biol. Rev. 67, 16-37 (2003).

11. Hwang, H. H., Yu, M. \& Lai, E. M. Agrobacterium-mediated plant transformation: biology and applications. Arabidopsis Book 15, e0186 (2017).

12. Mao, Y. F., Botella, J. R., Liu, Y. G. \& Zhu, J. K. Gene editing in plants: progress and challenges. Natl Sci. Rev. 6, 421-437 (2019).

13. Yang, X. et al. Plant biosystems design research roadmap 1.0. BioDesign Res. 2020, 8051764 (2020).

14. Harrison, S. J. et al. A rapid and robust method of identifying transformed Arabidopsis thaliana seedlings following floral dip transformation. Plant Methods 2, 19 (2006). 
15. Bhalla, P. L. \& Singh, M. B. Agrobacterium-mediated transformation of Brassica napus and Brassica oleracea. Nat. Protoc. 3, 181-189 (2008).

16. Shimizu, A., Shiratori, I., Horii, K. \& Waga, I. Molecular evolution of versatile derivatives from a GFP-like protein in the marine copepod Chiridius poppei. PLOS ONE 12, e0181186 (2017).

17. Chin, D. P. et al. Generation of brilliant green fluorescent petunia plants by using a new and potent fluorescent protein transgene. Sci. Rep. 8, 16556 (2018).

18. Sannigrahi, P., Ragauskas, A. J. \& Tuskan, G. A. Poplar as a feedstock for biofuels: a review of compositional characteristics. Biofuel Bioprod. Bior 4, 209-226 (2010).

19. Liu, Y. Q., Heying, E. \& Tanumihardjo, S. A. History, global distribution, and nutritional importance of Citrus fruits. Compr. Rev. Food Sci. F. 11, 530-545 (2012).

20. Yamasaki, S. et al. Arabidopsis thaliana cold-regulated 47 gene 5'-untranslated region enables stable high-level expression of transgenes. J. Biosci. Bioeng. 125, 124-130 (2018).

21. Matsui, T., Sawada, K., Takita, E. \& Kato, K. The longer version of Arabidopsis thaliana heat shock protein 18.2 gene terminator contributes to higher expression of stably integrated transgenes in cultured tobacco cells. Plant Biotechnol. 31, 191-194 (2014).

22. Gutierrez-Valdes, N. et al. Hairy root cultures-A versatile tool with multiple applications. Front. Plant Sci. 11, 33 (2020).

23. Gomes, C., Dupas, A., Pagano, A., Grima-Pettenati, J. \& Paiva, J. A. P. Hairy root transformation: a useful tool to explore gene function and expression in Salix spp. recalcitrant to transformation. Front. Plant Sci. 10, 1427 (2019).

24. Ma, Y. et al. Molecular analysis of rice plants harboring a multi-functional TDNA tagging system. J. Genet Genom. 36, 267-276 (2009).

25. Ye, X. D. et al. Enhanced production of single copy backbone-free transgenic plants in multiple crop species using binary vectors with a pRi replication origin in Agrobacterium tumefaciens. Transgenic Res. 20, 773-786 (2011).

26. Maher, M. F. et al. Plant gene editing through de novo induction of meristems. Nat. Biotechnol. 38, 84-89 (2020).

27. Breyer, D., Kopertekh, L. \& Reheul, D. Alternatives to antibiotic resistance marker genes for in vitro selection of genetically modified plants - scientific developments, current use, operational access and biosafety considerations. Crit. Rev. Plant Sci. 33, 286-330 (2014).

28. Verma, S. S., Chiinnusarny, V. \& Bansal, K. C. A simplified floral dip method for transformation of Brassica napus and B. carinata. J. Plant Biochem Biot. 17 197-200 (2008)
29. Liu, Z. Q. et al. Systematic comparison of $2 A$ peptides for cloning multi-genes in a polycistronic vector. Sci. Rep. 7, 2193 (2017)

30. Yuan, $G$. et al. Biosystems design to accelerate $C_{3}$-to-CAM progression. BioDesign Res. 2020, 3686791 (2020)

31. Hernandez-Garcia, C. M. \& Finer, J. J. Identification and validation of promoters and cis-acting regulatory elements. Plant Sci. 217, 109-119 (2014).

32. Notaguchi, M. \& Okamoto, S. Dynamics of long-distance signaling via plant vascular tissues. Front. Plant Sci. 6, 161 (2015).

33. Dietz-Pfeilstetter, A. Stability of transgene expression as a challenge for genetic engineering. Plant Sci. 179, 164-167 (2010).

34. Rigoulot, S. B. et al. Imaging of multiple fluorescent proteins in canopies enables synthetic biology in plants. Plant Biotechnol. J. 19, 830-843 (2021)

35. Xie, M. et al. A 5-enolpyruvylshikimate 3-phosphate synthase functions as a transcriptional repressor in Populus. Plant Cell 30, 1645-1660 (2018).

36. Yuan, $G$ et al. PROTEIN PHOSHATASE 2A B'a and $\beta$ maintain centromeric sister chromatid cohesion during meiosis in. Arabidopsis. Plant Physiol. 178, 317-328 (2018).

37. Li, X. Infiltration of Nicotiana benthamiana protocol for transient expression via Agrobacterium. Bio-Protoc. 1, e95 (2011).

38. Chen, L. Z. et al. A method for the production and expedient screening of CRISPR/Cas9-mediated non-transgenic mutant plants. Hortic. Res. 5, 13 (2018).

39. Filichkin, S. A. et al. Alcohol-inducible gene expression in transgenic. Populus. Plant Cell Rep. 25, 660-667 (2006).

40. Weigel, D. \& Glazebrook, J. Quick miniprep for plant DNA isolation. Cold Spring Harb. Protoc. 2009, pdb.prot5179 (2009).

41. Wang, Y. et al. Validation of reference genes for gene expression by quantitative real-time RT-PCR in stem segments spanning primary to secondary growth in Populus tomentosa. Plos ONE 11, e0157370 (2016).

42. Lu, H. W. et al. RNA interference suppression of AGAMOUS and SEEDSTICK alters floral organ identity and impairs floral organ determinacy, ovule differentiation, and seed-hair development in Populus. New Phytologist 222, 923-937 (2019).

43. Lu, H. W., Gordon, M. I., Amarasinghe, V. \& Strauss, S. H. Extensive transcriptome changes during seasonal leaf senescence in field-grown black cottonwood (Populus trichocarpa Nisqually-1). Sci. Rep. 10, 6581 (2020).

44. Qiao, Z. \& Libault, M. Unleashing the potential of the root hair cell as a single plant cell type model in root systems biology. Front. Plant Sci. 4, 484 (2013).

45. Zhang, F., LeBlanc, C., Irish, V. F. \& Jacob, Y. Rapid and efficient CRISPR/Cas9 gene editing in Citrus using the YAO promoter. Plant Cell Rep. 36, 1883-1887 (2017). 